\title{
Adaptive Vibrotactile Threshold Estimation of the Glabrous Hand And Perioral Face Following MCA Stroke
}

\author{
Steven M Barlow ${ }^{1 *}$, Rebecca Custead ${ }^{1}$ and Jaehoon Lee ${ }^{2}$ \\ ${ }^{1}$ Department of Special Education and Communication Disorders and Biological Systems Engineering, USA
}

${ }^{2}$ Department of Educational Psychology \& Leadership, USA

*Corresponding author: Steven M Barlow, Department of Special Education and Communication Disorders and Biological Systems Engineering, USA

\section{ARTICLE INFO}

Received: 幽 November 22, 2019

Published: December 02, 2019

Citation: Steven M Barlow, Rebecca Custead, Jaehoon Lee. Adaptive Vibrotactile Threshold Estimation of the Glabrous Hand And Perioral Face Following MCA Stroke. Biomed J Sci \& Tech Res 23(3)2019. BJSTR. MS.ID.003899.

Keywords: Stroke; Somatosensory; Automatic Adaptive Cutaneous Threshold Tracking; Glabrous; Nonglabrous

Abbreviations: VDT: Vibrotactile Detection Thresholds, MCA: Middle Cerebral Artery, FMA: Fugl Meyer Assessment, UE: Upper Extremity, DVRT: Differential Variable Reluctance Transformer, FUS: Focused Ultrasound

\section{ABSTRACT}

Purpose: Cerebrovascular stroke often manifests movement disorders, while changes in somatosensory function may be inconspicuous and difficult to measure. A comprehensive movement disorder evaluation would benefit from an assessment of the somatosensory system to highly controlled vibrotactile stimulation over a range of frequencies.

Method: An automatic single-interval up/down adaptive procedure using an embedded field programmable gate array microcontroller for stimulus control and response logging was used to estimate Vibrotactile Detection Thresholds (VDT) bilaterally for the glabrous index finger and perioral hairy skin at the oral angle in a cohort of 7 unilateral Middle Cerebral Artery (MCA) stroke survivors in response to vibrotactile stimuli presented at 5, 10, 50,150, 250, and $300 \mathrm{~Hz}$. Linear mixed modeling revealed significant main effects for stroke status, structure and vibrotactile frequency. A secondary analysis compared ipsilesional/contralesional structures with 89 sets of neurotypical thresholds from our laboratory database.

Results: VDTs were dependent on test frequency $(p<.0001$, Cohen's $d=0.45$ 1.15), with VDTs significantly higher for the oral angle condition compared to the finger condition $(\mathrm{p}<.0001, \mathrm{~d}=0.43)$. Stroke patients had significantly higher VDTs than normal adults $(\mathrm{p}<.0001, \mathrm{~d}=0.62)$. The secondary analyses revealed significant differences between the neurotypical oral angle VDTs and both the contralesional $(\mathrm{p}<$ $.01, \mathrm{~d}=0.64)$ and ipsilesional oral angle VDTs $(\mathrm{p}<.05, \mathrm{~d}=0.56)$. Larger differences were found between the neurotypical glabrous index finger VDTs and the contralesional index finger $(p<.0001, d=1.45)$. Finally, the contralesional index finger manifest significantly higher VDTs than the ipsilesional index finger $(\mathrm{p}<.0001, \mathrm{~d}=1.13)$.

Conclusion: Our automated adaptive VDT tracking algorithm provides clinicians with a reliable tool for rapid assessment of the cutaneous somatosensory system on both glabrous and hairy skin in stroke survivors to assess neurosensory status.

\section{Introduction}

The skin of the face and hand possess unique histologic features and can undergo conformational changes due to muscle contractions to convey our identity and feelings, communicate with speech and nonverbal facial gesture, or interact and manipulate the environment. The skin also serves as a receiver of haptic information for detection and recognition of touch, shape, texture, pleasure, movement, and to alert us of potential environmental hazards [1]. In many acquired and progressive diseases however, somatosensory impairment often leads to higher mortalities due to limited activity and increased risk of injury. Not only is there a disruption of tactile reception and interpretation, there is often large deficit in coordinated motor performance due to a loss of feedback mechanisms necessary for stereognosis, kinesthesia, and movement related proprioception. For example, the modulation 
of precision grip by anticipatory vibrotaction during $100 \mathrm{~Hz}$ and $250 \mathrm{~Hz}$ stimulation provides important evidence on the role of mechanoreception to influence motor reflex action during voluntary movement [2]. Similarly, accurate orofacial haptic processing is required for intelligible speech, oral continence and emotion-congruent facial expression [3].

An estimated one in two stroke survivors have enough loss of the sense of touch that they have difficulty with everyday activities such as eating, dressing, transferring and communicating needs $[4,5]$. Ischemic damage to cortical and subcortical regions in stroke results in a loss of top-down control of motor execution, and degraded management of sensory signals from peripheral receptors $[6,7]$. Additionally, there is widespread disruption of excitatory and inhibitory networks responsible for somatosensory, executive and visuospatial processing $[8,9]$. The resulting impairment can lead to life-long sensory sequelae and contributes to functional deficit and hemispatial neglect $[10,11]$. Particularly in stages of post-injury motor-speech rehabilitation, somatosensory deficit or improvement can be difficult to identify in many of these patients.

Despite the importance of assessing the integrity of somatosensory pathways in brain injury and disease, access to non-invasive and time-efficient methods are limited. In clinical settings it can be challenging to evaluate specific somatosensory impairment or ascertain how much somatosensory damage is contributing to problems with motor performance $[12,13]$. From a long-term care perspective, compromised somatosensory function is related to longer length of stays for institutionalized patients $[14,15]$, and lower quality of life ratings from patients who are living at home Baum [16].

Thus, the goal of the present study was to assess finger and lower face vibrotactile detection thresholds in adults who have sustained a unilateral Middle Cerebral Artery (MCA) stroke and compare with same measures obtained from a cohort of neurotypical adults. Frequently overlooked in many standardized speech, language and gross motor assessments, deficits in haptic processing can result in an impaired capacity to regulate, organize and modulate communicative responses. In our cohort of stroke survivors, we hypothesized our VDT adaptive procedure would reveal significant differences in vibrotactile threshold as a function of site (hand vs face), stimulus frequency and stroke status (normal vs MCA stroke).

\section{Method}

\section{Participants}

Seven hemiparetic male stroke survivors participated in this study (mean age $=46.71$ years, $S D=20.14$ ). Inclusion criteria: chronic cerebrovascular infarct ( $>6$ months) primarily affecting sensorimotor function, unilateral stroke in the territory of the MCA as confirmed by medical record and high-resolution anatomical MRI, $20 / 20$ vision, ability to follow instructions and perform a button press. Exclusion criteria: total paralysis of contralesional limb or face, dementia, aphasia. Gross motor impairment was evaluated in each participant using the Fugl-Meyer Assessment Upper Extremity (FMA-UE), [17,18] prior to vibrotactile assessment (Table 1).

Table 1: Stroke participant profiles.

\begin{tabular}{|c|c|c|c|c|c|c|}
\hline ID & Age & Gender & Lesion Territory & Months Post-stroke & \multicolumn{2}{|c|}{ Fugl-Meyer UE Motor Function Score (66 max) } \\
\hline & & & & & R & L \\
\hline 1 & 67 & M & L MCA & 70 & 59 & 66 \\
\hline 2 & 31 & M & L MCA & 37 & 60 & 65 \\
\hline 3 & 23 & M & L MCA & 143 & 34 & 66 \\
\hline 4 & 47 & M & L MCA & 38 & 38 & 24 \\
\hline 5 & 66 & M & R MCA & 87 & 66 & 33 \\
\hline 6 & 26 & M & R MCA & 30 & 66 & 66 \\
\hline 7 & 67 & M & L MCA & 86 & 6 \\
\hline
\end{tabular}

In addition to comparing VDTs between the ipsilesional and contralesional skin sites for stroke survivors, we also compared their thresholds with an existing database of neurotypical VDTs sampled in our laboratory from eighty-nine adults (59F/30M [24.33 ( $S D=5.68)$ years]. Eighty-six of these neurotypical adults reported right-hand dominance, and 3 reported left-hand dominance. Inclusion criteria: no report of neurological or psychiatric illness, and no scheduled medication. Exclusion criteria: neurological, motor control deficits, psychiatric abnormalities, trauma to face and/or hand, or abnormal skin sensitivity on face or hand. All participants were recruited regardless of race or ethnicity, and written informed consent, approved by the university Institutional Review Board, was obtained.

\section{Vibrotactile Detection Threshold (VDT) Assessment for Hand and Face}

Stimulus control. A linear electrodynamic motor (Brüel \& Kjaer model 4810 Minishaker, +/- 3mm range) controlled by our software (VIBROS) was used to assess cutaneous vibrotactile sensitivity in the lower face and hand [19,20]. Adaptive stimulus control was achieved using a National Instruments cRIO real-time FPGA embedded controller programmed in LabVIEW to synthesize (NI 
9263, 16-bit, $100 \mathrm{KS} / \mathrm{s}$ ) 1-second sinusoidal bursts followed by a 1-second off-state. A linear rise-fall decay function of $100 \mathrm{~ms}$ during burst generation circumvented mechanical transients. This voltage signal was conditioned by a Brüel \& Kjaer model 2706 power amplifier and input to the motor. The Minishaker includes custom fixtures and an integral Schaevitz subminiature Differential Variable Reluctance Transformer (DVRT) sensor to transduce displacement for precision vibrotactile stimulation and measurement, nylon contactor probe (Area $=0.5 \mathrm{~cm}^{2}$ ), and a stainless-steel rigid surround (annular gap = $1 \mathrm{~mm}$ ). The surround was coupled to a linear micrometer translation stage that was used for actuator displacement calibration and skin contactor preload. This fixture configuration allows the surface of the rigid surround to be adjusted relative to the contactor probe to produce a $500 \mu \mathrm{m}$ tissue preload against the moving stimulator probe. The DVRT displacement sensor provided an output signal linearly related to contactor probe displacement from DC to $800 \mathrm{~Hz}$.

Participants were seated in a medical examination chair with an articulating headrest and a height-adjustable worktable and asked to press a response button as soon as they detected the vibratory stimulus. If needed, the participant's tested arm was rested on a pillow for stability. The orientation of the motor's probe-surround to the glabrous finger and oral angle is shown in Figure 1. A double adhesive collar (7/16" ID) was placed on the stainless-steel surround fixture of the Minishaker to secure placement of the probe on the skin.

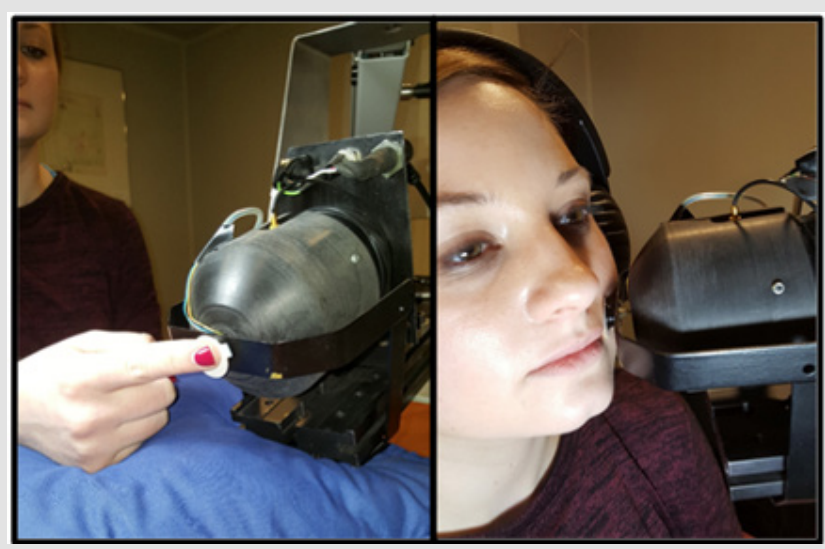

Figure 1: VDT (Vibrotactile detection thresholds) at the glabrous index fingertip and oral angle.

\section{Adaptive Vibrotactile Threshold Tracking Algorithm}

A Single-Interval Up/Down (SIUD) adaptive procedure, described by Lecluyse and Meddis [21] for assessing auditory function, was adapted to estimate vibrotactile thresholds at 5, 10, 50, 150,250 , and $300 \mathrm{~Hz}$ on the glabrous surface of the distal phalanx of the index finger, and at nonglabrous surface of the oral angle. Test order for site and stimulus frequency was randomized among participants. Narrow-band noise [66-68 dB(C) SPL] was presented to each participant through circumaural headphones during vibrotactile threshold tracking to mask the acoustic emittance associated with the Minishaker at test frequencies $\geq 50 \mathrm{~Hz}$
Participants were instructed to press a response button when they 'felt' the vibratory stimulus. The initial stimulus amplitude for any given stimulus frequency was set at a supra-threshold level in order to ensure a detection response. The initial step size was set at $10 \mathrm{~dB}$, and then randomly varied in a $\pm 5 \mathrm{~dB}$ range relative to the initial amplitude. After the first negative response, the stimulus level was set at the mid-point between the previous 2 levels, and a 2-dB step was subsequently utilized. The VDT test procedure continued for 8 trials starting from the trial prior to the first negative response. The algorithm implemented in this study also used false positive detection tests (foils) in which no vibrotactile stimulus was presented to ensure participant vigilance. These false positive trials were implemented in $20 \%$ of the successive trials, and on detection of a false positive trial, it was discarded, and a new trial was restarted. The number of trials $(n=8)$ included in threshold estimation was chosen in order to attain an accuracy of $\pm 2 \mathrm{~dB}$ and this number excludes the false positive trials which typically extend any given threshold run by 1 or 2 additional trials [19].

\section{Statistical Analysis}

Linear mixed modeling was completed to compare vibrotactile threshold between frequency $(5,10,50,150,250,300 \mathrm{~Hz})$, structure (oral angle, finger), and stroke status (normal, stroke) conditions. An alternative classification of structure comparing neurotypical (dominant side) and stroke structures was applied in a subsequent model (i.e., normal oral angle, normal finger, contralesional oral angle, ipsilesional oral angle, contralesional finger, and ipsilesional finger). The models accounted for participants' age and sex as well as nesting of repeated measurements within participants, thereby providing unbiased estimates for condition differences. When an overall difference was significant across conditions, adjusted means were pairwise compared at a Bonferroni-corrected alpha level while controlling for Type I error at the nominal level. A proper error covariance structure was determined based on model fit (i.e., adjusted Akaike Information Criterion, Bayesian Information Criterion). All statistical analyses were conducted using SAS 9.4 (SAS Institute) [22].

\section{Results}

The estimated marginal means and standard errors of vibrotactile threshold adjusted for age and sex in the mixed modeling are shown in Figure 2. Bonferroni pairwise comparisons indicated that vibrotactile threshold was significantly higher in the 5 and $10 \mathrm{~Hz}$ conditions than in other frequency conditions (all $\mathrm{p}<.0001$, Cohen's $d=0.45-1.15$ ), and significantly higher in the $5 \mathrm{~Hz}$ condition compared to the $10 \mathrm{~Hz}$ condition $(\mathrm{p}<.0001, \mathrm{~d}=$ 0.61 ). In addition, vibrotactile threshold was significantly higher for the oral angle condition compared to the finger condition ( $p<.0001, d=0.43$ ). It was found that stroke patients had significantly higher VDTs than normal adults $(\mathrm{p}<.0001, \mathrm{~d}=0.62)$. The secondary mixed modeling and Bonferroni pairwise analyses comparing neurotypical and stroke structures revealed significant differences between the neurotypical oral angle VDTs and both the 
contralesional $(\mathrm{p}<.01, \mathrm{~d}=0.64)$ and ipsilesional oral angle VDTs $(p<.05, d=0.56)$. Even larger differences and corresponding effect sizes were found between the neurotypical glabrous index finger VDTs and the contralesional index finger $(\mathrm{p}<.0001, \mathrm{~d}=1.45)$. Finally, the contralesional index finger manifest significantly higher VDTs than the ipsilesional index finger in our stroke cohort ( $p<$ $.0001, \mathrm{~d}=1.13$ ).

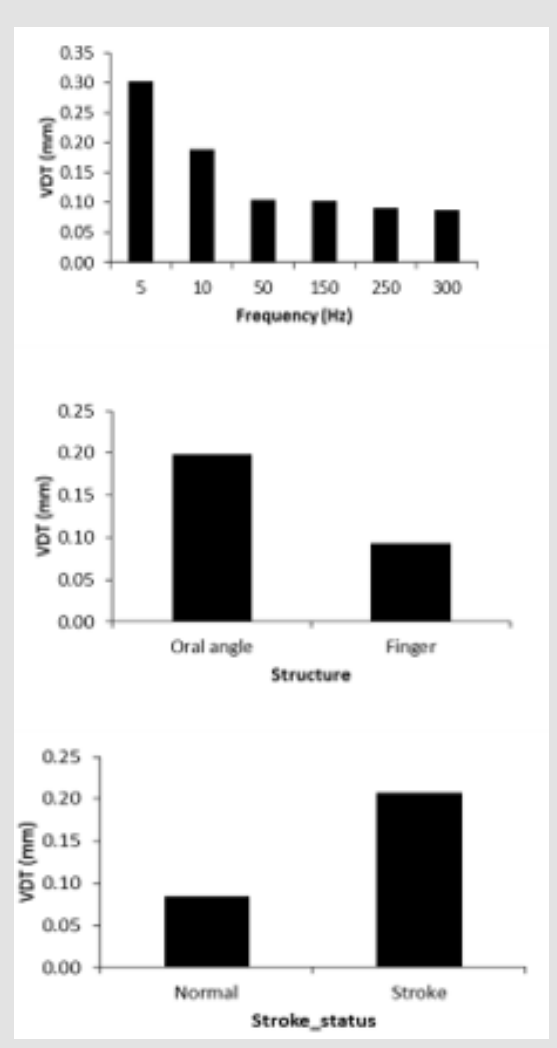

Figure 2: Estimated marginal means for main effects, including vibrotactile stimulus frequency, somatic structure, and stroke status.

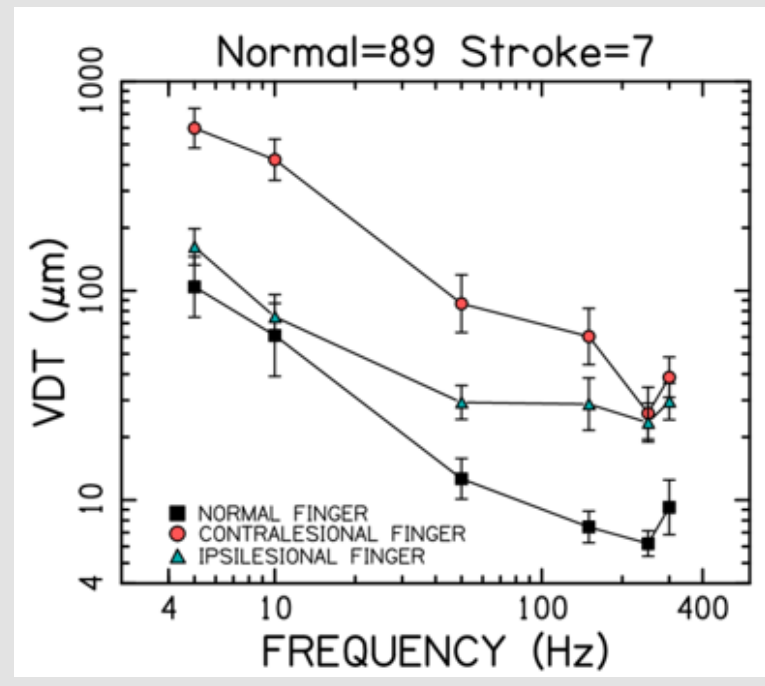

Figure 3: Vibrotactile threshold functions for the P4 segment of the glabrous index finger among normal (dominant) and stroke participants (contra- vs. ipsilesional). Error bars $=$ SEM.
Index finger VDTs $(\mathrm{M} \pm \mathrm{SEM})$ for the stroke participants plotted on a log-log scale relative to our database of neurotypical tactile thresholds are shown in Figure 3. The significant difference between contralesional and ipsilesional index finger VDTs is readily apparent, especially for test frequencies spanning $5 \mathrm{~Hz}$ through $150 \mathrm{~Hz}$. This plot also shows the contralesional finger VDTs among our stroke participants to be nearly an order of magnitude greater than the normal VDTs for this glabrous skin surface throughout the range of stimulus frequencies, with some preservation of the $250 \mathrm{~Hz}$ notch ascribed to the Pacinian corpuscle's best frequency response.

Oral angle VDTs in response to stimulation of the hairy skin near the corner of the mouth $(\mathrm{M} \pm \mathrm{SEM})$ for the stroke participants plotted relative to our neurotypical database is shown in Figure 4. A significant difference is apparent between contralesional and ipsilesional oral angle VDTs for test frequencies $5 \mathrm{~Hz}$ through 150 Hz. This plot also shows the contralesional oral angle VDT function is elevated more than 2-fold throughout this frequency range. Note the absence of the classic PC dip in the oral angle VDT function which is consistent with the lack of Pacinian corpuscle mechanoreceptors in the lower face.

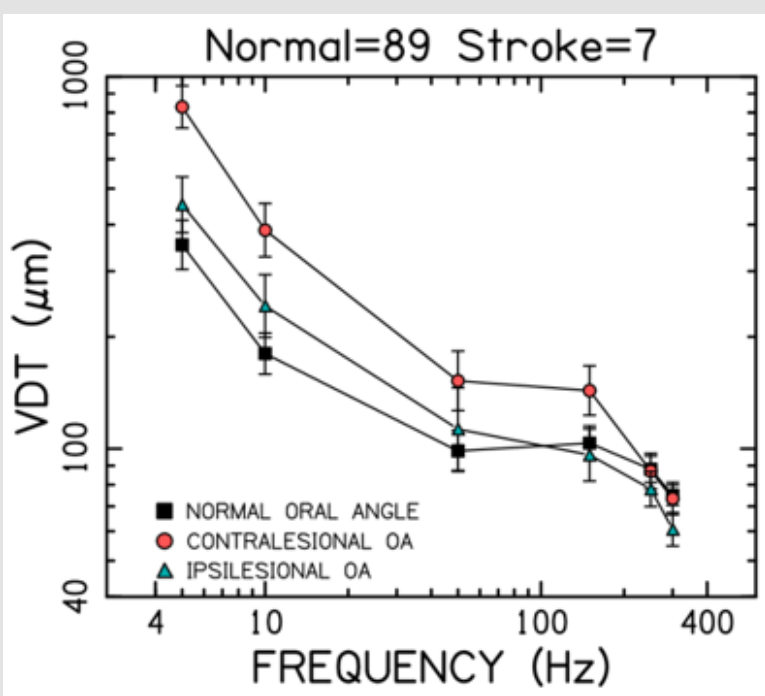

Figure 4: Vibrotactile threshold functions for the oral angle (OA) hairy skin among normal (dominant) and stroke participants (contra- vs, ipsilesional). Error bars = SEM.

\section{Discussion}

Vibrotactile sensitivity of the hairy skin at the oral angle and the glabrous surface of the distal (P4) phalanx of the glabrous index finger was assessed in a cohort of MCA stroke survivors using an application we developed (VIBROS) based on an automated SIUD adaptive threshold estimation procedure [19-21]. Highly significant contralesional deficits in vibrotactile sensitivity was found for the glabrous index finger and hairy skin near the corner of the mouth among the stroke survivors tested. There were no significant differences for hand or face VDTs as a function of sex which is consistent with previously published studies $[19,23,24]$. 
The use of 6 test frequencies provides the clinician a comprehensive profile of vibrotactile detection for contralesional and ipsilesional skin contrasting slow adapting and rapid adapting mechanoreceptor performance in the vibrogram. Comparison to our normative database also sheds additional light on the absolute VDT levels between these two groups of participants. The 4-minute adaptive SIUD VDT procedure used in the present study is efficient and practical for clinical use with stroke survivors or impaired populations. Fewer trials are required to yield thresholds similar to those attained by traditional methods like the two-interval forcedchoice, two-down/one-up and maximum-likelihood procedures, permitting maximal precision for repeated comparisons of clinical groups. The number of trials used for VDT estimation in this study $(\mathrm{n}=8)$ is in addition to programmed false positive or 'catch' trials and based on the Lecluyse and Meddis (2009) mathematical model this VDT estimation yields $\pm 2 \mathrm{~dB}$ accuracy.

\section{Contrasting Cutaneous Sensitivity of the Hand and Face}

The differences in vibrotactile sensitivity for the hand $[25,26]$ and face $[19,26,27]$ are consistent with known differences in the representation of $A \beta$ mechanoreceptor typing and integument and are confirmed in the present report using the SIUD adaptive threshold protocol in stroke survivors and normal controls. The classic Pacinian U-shaped response characteristic in the glabrous hand for vibratory input at $250 \mathrm{~Hz}$ is absent in perioral hairy skin [28-30]. This is consistent with histological and physiological studies of facial skin which have not found PC receptors in facial skin [3133]. From a speech pathology, rehabilitative standpoint, many facial muscle fibers insert directly into the skin rather than the connective tissue making it possible for embedded mechanoreceptors such as pseudo-Ruffini endings to encode proprioceptive information about changes in muscle length and force required for intelligible speech and facial gesture [29,34,35]. Conformational changes to skin during imposed or voluntary movements also results in mechanoreceptor activity and a stream of somatosensory flow along peripheral nerves to the central nervous system [34]. Anatomical and morphological differences between the hand and face are evident. Thus, information on somatosensory status would be useful for determining the prognosis or designing treatments for neurological insults (e.g., cerebral stroke) or diseases common in older adults $[36,37]$.

\section{Future Applications for Neurorehabilitation Utilizing Vibrotactile Sensing to Advance Haptic Technologies}

Until recently, most methods for evaluating sensory competence in the clinical setting have been based on user-friendly, historical methods such as self-report of light touch with specially designed filaments, two-point discrimination, and thermal sensation measures. These can have variable results and reveal only gross deficit particularly in very young or advanced-aged patients [3840]. Newer, device-based methods can be lengthy, require specialty training and equipment for administration and interpretation, and are rarely easily portable [13,41-43].

An intact trigeminal-lemniscal-thalamocortical pathway system is required to encode and transmit mechanosensory information from the lower face to somatosensory cortex. Similarly, top-down regulatory feedback from cortical centers are needed to modulate skilled orofacial movements such as speech, oral continence, swallow and facial gesture. Often adversely affected in MCA stroke, sensorimotor impairment can prevent maximum benefit of rehabilitation $[44,45]$. Vibrotaction plays a key role in the discrimination of temporal and rhythmic aspects of speech and can restrict errors that contribute to poor intelligibility after stroke $[46,47]$. This implies that a reliable, short-duration measure of post-injury sensation such as the one described in this study should be included in basic rehabilitative assessments.

With the emergence of more advanced somatosensory assessments, brain stimulation techniques guided by tactile sensing technologies show promise in neurotherapeutics [48-50]. For example, some investigators are exploring the effects of transcranial direct current stimulation on neuromodulation of primary somatosensory cortex on VDT detection and somatosensory discrimination [51]. Focused Ultrasound (FUS) sonication of S1 has been used to evoke both sonication-specific Electroencephalographic (EEG) responses and various tactile sensations from the hand area of the postcentral gyrus [52]. Others have used mapping of local field potentials to elucidate the functional properties of cortical areas involved in multimodal processing of somatosensory inputs, such as the posterior insula, is regarded as the so-called 'ouch-zone' and presumed to play a key role pain perception. Direct intracerebral recordings, however, have shown that painful and nonpainful stimuli $(250 \mathrm{~Hz}$ vibration of index finger) elicit very similar responses throughout the human insula [53].

Artificial sensory feedback systems for both face and limb is a rapidly emerging technology which incorporates small vibrating motors or tactors, placed at different parts of the body, to provide spatial as well as temporal feedback to compensate for lost proprioception in the limbs [54]. Vibrotactile feedback has demonstrated efficacy in applications to individuals with lowerlimb amputations, vestibular impairments, age-related loss of balance, speech motor control and the Tadoma method for speech reception [55-58]. Looking toward these exciting prospects in the future, we note that a repeated potential drawback to stroke and somatosensory research is the high amount of variability across participants and disease states. A common thread in study limitations of this type include changes in touch-vibrotactile sensitivity, cognition, as well as orofacial and limb motor performance across the lifespan. Additionally, the pathophysiology of sensorimotor damage in stroke undoubtedly play a role in diminution of vibrotactile sensitivity. Factors such as smoking, 
hyperlipidemia, hypertension, type I and II diabetes and neural hypoxia likely affect somatosensory pathways and blood supply to both peripheral and neural tissues [59-61].

\section{Conclusion}

Our study demonstrated that an automated SIUD adaptive threshold tracking procedure can assess vibrotactile sensitivity for the hand and face in adult stroke survivors in a time-efficient manner to provide a mechanosensory threshold profile over a range of six test frequencies $(5-300 \mathrm{~Hz}$ ) for contralesional and ipsilesional skin sites in the hands and face. This SIUD procedure replicated previous findings in neurotypical young adults showing significant main effects for stimulation site and stimulus frequency presumably due to the differences in the density and type of mechanoreceptors innervating the face and glabrous hand.

The incidence of brain injury related to cerebrovascular stroke increases with age, and the sense of touch is altered in older adults $[62,63]$. Automated VDT testing can be used to diagnose the extent of sensory impairment, monitor the progress of the disease or injury, and has the potential to monitor the effectiveness of the treatment utilized. Functional neuroimaging studies that correlate the tactile perception with the neural response are needed to further elucidate these differences in health and disease $[37,64]$. This information would enable us to develop better diagnosis and treatment design for neurological disorders and diseases such as cerebrovascular stroke that are prevalent in older adults.

\section{Acknowledgment}

Special gratitude is expressed to Chelsey Krug, Kelsey Sestak, AnnaJean Scarborough, and Doug Kieweg for assistance with data collection in this study.

\section{References}

1. Arda 0 , Göksügür N, Tüzün Y (2014) Basic histological structure and functions of facial skin. Clinics in Dermatology 32(1): 3-13.

2. Okamoto S, Wiertlewski M, Hayward V (2016) Anticipatory Vibrotactile Cueing Facilitates Grip Force Adjustment during Perturbative Loading. IEEE Transactions on Haptics 9: 233-242.

3. Spape M, Harjunen V, Ravaja N (2017) Effects of touch on emotional face processing: A study of event-related potentials, facial EMG and cardiac activity. Biological Psychology 124: 1-10.

4. Holland A, Frattali C, Fromm D (1999) Communication Activities of Daily Living: CADL-2. Pro-Ed.

5. Carey L, Lamp G, Turville M (2016) The state-of-the-science on somatosensory function and its impact on daily life in adults and older adults and following stroke: a scoping review. OTJR: Occupation, Participation and Health 36(2): 275-415.

6. Kim J (2007) Patterns of sensory abnormality in cortical stroke: evidence for a dichotomized sensory system. Neurology 68(3): 174-180.

7. Mah Y, Husain M, Rees G, Nachev P (2014) Human brain lesion-deficit inference remapped. Brain 137: 2522-2531.

8. Kenzie J, Semrau J, Findlater S, Yu A, Desai J, et al. (2016) Localizing of impaired kinesthetic processing in post-stroke. Frontiers in Human Neuroscience 10: 505 .
9. Meyer S, De Bruyn N, Lafosse C, Van Dijk M, Michielsen M, et al. (2016) Somatosensory Impairments in the Upper Limb Poststroke: Distribution and Association with Motor Function and Visuospatial Neglect. Neurorehabilitation and Neural Repair 30(8): 731-742.

10. Nijboer T, Van de port I, Schepers V, Post M, Visser Meily A (2013) Predicting functional outcome after stroke: the influence of neglect on basic activities in daily living. Frontiers Human Neuroscience 7: 182.

11. Torre K, Hammami N, Metrot J, van Dokkum L, Coroian F (2013) Somatosensory- related limitation for bimanual coordination after stroke. Neurorehabilitation and Neural Repair 27(6): 507-515.

12. Smania N, Montagnana B, Faccioli S, Fiaschi A, Aglioti S (2003) Rehabilitation of somatic sensation and related deficit of motor control in patients with pure sensory stroke. Archives Physical Medical Rehabilitation 84(11): 1692-1702.

13. Connell L, Lincoln N, Radford K (2008) Somatosensory impairment after stroke: frequency of different deficits and their recovery. Clinical Rehabilitation 22(8): 758-767.

14. Winward C, Halligan P, Wade D (1999) Current practice and clinical relevance of somatosensory assessment after stroke. Clinical Rehabilitation 13(1): 48-55.

15. Sommerfield D, von Arbin M (2004) The impact of somatosensory function on activity performance and length of hospital stay in geriatric patients with stroke. Clinical Rehabilitation 18(2): 149-55

16. Baum C (2011) Fulfilling the promise: Supporting participation in daily life. Archives Physical Medicine and Rehabilitation 92(2): 169-175.

17. Fugl Meyer AR, Jääskö L, Leyman I, Olsson S, Steglind S (1975) The poststroke hemiplegic patient. 1. A method for evaluation of physical performance. Scandinavian Journal Rehabilitative Medicine 7(1): 13-31.

18. Duncan PW, Propst M, Nelson SG (1983) Reliability of the Fugl-Meyer assessment of sensorimotor recovery following cerebrovascular accident. Physical Therapy 63(10): 1606-1610.

19. Venkatesan L, Barlow SM, Kieweg D (2015) Age- and sex-related changes in vibrotactile sensitivity of hand and face in neurotypical adults. Somatosensory Motor Research 32(1): 44-50.

20. Barlow SM, Custead R (2019) Vibrography: Single-Interval Up/Down (SIUD) adaptive vibrotactile threshold estimation of the glabrous hand and perioral face in neurotypical adults. Biomedical J Scientific \& Technical Research 22(4)-2019.

21. Lecluyse W, Meddis R (2009) A simple single-interval adaptive procedure for estimating thresholds in normal and impaired listeners. Journal Acoustical Society America 126(5): 2570-2579.

22. SAS Institute. SAS/STAT 9.4 user's guide. Cary, NC: SAS Institute Inc; 2002-2012.

23. Verrillo RT (1979) Comparison of vibrotactile threshold and suprathreshold responses in men and women. Perception \& Psychophysics 26(1): 20-24.

24. Gescheider GA, Bolanowski SJ, Hall KL, Hoffman KE, Verrillo RT (1994) The effects of aging on information-processing channels in the sense of touch: I. Absolute sensitivity. Somatosensory Motor Research 11(4): 345-357.

25. Verrillo RT (1983) Vibrotactile subjective magnitude as a function of hand preference. Neuropsychologia 21(4): 383-395.

26. Barlow SM (1987) Mechanical frequency detection thresholds in the human face. Experimental Neurology 96(2): 253-261.

27. Andreatta RD, Davidow JH (2006) Mechanical frequency and stimulationsite-related differences in vibrotactile detection capacity along the lip vermilion in young adults. Clinical Oral Investigations 10(1): 17-22.

28. Johansson RS, Trulsson M, Olsson KA, Westberg KG (1988) Mechanoreceptor activity from the human face and oral mucosa. Experimental Brain Research 72(1): 204-208. 
29. Nordin M, Hagbarth KE (1989) Mechanoreceptive units in the human infra-orbital nerve. Acta Physiologica Scandinavia 135(2): 149-161.

30. Kent RD, Martin RE, Sufit RL (1990) Oral sensation: A review and clinical prospective. In H. Winitz (Eds.), Human Communication and its Disorders. Norwood. NJ: Apex Press.

31. Dubner R, Sessle BJ, Storey AT (1978) Touch. In Ronald Dubner (Eds.), The Neural Basis of Oral and Facial Function. New York Pp. 74-107.

32. Munger BL, Halata Z (1983) The sensory innervation of primate facial skin I Hairy skin. Brain Research 286: 45-80.

33. Halata Z, Munger BL (1983) The sensory innervation of primate facial skin. II. Vermilion border and mucosal lip. Brain Research 286(1): 81107.

34. Barlow SM (1998) Real-time modulation of speech-orofacial motor performance by means of motion sense. Journal Communication Disorders 31(6): 511-534.

35. Huertas Hoyas E, Pedrero Pérez E, Águila Maturana A, García LópezAlberca S (2015) Functionality predictors in acquired brain damage. Neurologia 30(6): 339-346.

36. Wohlert AB, Smith A (1998) Spatiotemporal stability of lip movements in older adult speakers. Journal Speech Language Hearing Research 41(1): 41-50.

37. Custead R, Oh H, Wang Y, Barlow SM (2017) Brain encoding of saltatory velocity through a pulsed pneumotactile array in the lower face. Brain Research 1677: 58-73.

38. Bell-Krotoski JA, Buford WL (1997) The force/time relationship of clinically used sensory testing instruments. J Hand Therapy 10(4): 297309.

39. Auld M, Johnston L (2016) A touchy topic: tactile assessment among pediatric therapists. Disability and Rehabilitation 40(3): 267-276.

40. Heft M, Robinson M (2017) Somatosensory function in old age. Journal Oral Rehabilitation 44(4): 327-332.

41. Lincoln N, Crow J, Jackson J, Waters G, Adams S, et al. (1991) The unreliability of sensory assessment. Clinical Rehabilitation 5: 273-282.

42. Wang Q, Markopoulos P, Yu B, Chen W, Timmermans A (2017) Interactive wearable systems for upper body rehabilitation: a systematic review. Journal Neuroengineering and Rehabilitation 14(1): 20.

43. Held J, Klaassen B, van Beijnum B, Luft A, Veltink P (2016) Usability evaluation of a vibrotactile feedback system in stroke subjects. Frontiers in Bioengineering and Biotechnology 4: 98.

44. Hollander M, Koudstaal P, Bots M, Grobbee D, Hofman A (2003) Incidence, risk, and case fatality of first ever stroke in the elderly population: The Rotterdam Study. Journal Neurology Neurosurgery Psychiatry 74: 317321.

45. Etter N, Dressler E, Andreatta R (2016) The relationship between labial vibrotactile detection and pure-tone hearing thresholds in healthy, ageing adults. International Journal Speech-Language Pathology 18(1): 89-96.

46. Fries W, Swihart A (1990) Disturbance of rhythm sense following right hemisphere damage. Neuropsychologia 28(12): 1317-1323.

47. Navarra J, Soto-Faraco S, Spence C (2014) Discriminating speech rhythms in audition, vision, and touch. Acta Physiologica 151: 197-205.

48. George MS, Aston Jones G (2010) Noninvasive techniques for probing neurocircuitry and treating illness: vagus nerve stimulation (VNS), transcranial magnetic stimulation (TMS) and transcranial direct current stimulation (tDCS). Neuropsychopharmacology 35: 301-16.

49. Hoy KE, Fitzgerald PB (2010) Brain stimulation in psychiatry and its effects on cognition. Nature Reviews Neurology 6(5): 267-275.

50. Lee W, Chung YA, Jung Y, Song IU, Yoo SS (2016) Simultaneous acoustic stimulation of human primary and secondary somatosensory cortices using transcranial focused ultrasound. BMC Neuroscience 17(1): 68.

51. Labbé S, Meftah EM, Chapman CE (2016) Effects of transcranial direct current stimulation of primary somatosensory cortex on vibrotactile detection and discrimination. Journal Neurophysiology 115(4): 19781987.

52. Lee W, Kim H, Jung Y, Song IU, Chung YA, et al. (2015) Imageguided transcranial focused ultrasound stimulates human primary somatosensory cortex. Science Reports 5: 8743.

53. Liberati G, Klöcker A, Safronova MM, Ferrão Santos S, et al. (2016) Nociceptive Local Field Potentials Recorded from the Human Insula Are Not Specific for Nociception. PLoS Biology 14(1): e1002345.

54. Leineweber MJ, Shi S, Andrysek J (2016) A Method for Evaluating Timeliness and Accuracy of Volitional Motor Responses to Vibrotactile Stimuli. Journal Visualized Experiments 2: 114.

55. Leotta D, Rabinowitz W, Reed C, Durlach NI (1988) Preliminary results of speech-reception tests obtained with the synthetic Tadoma system. Journal Rehabilitation Research and Development 25(4): 45-52.

56. Wentink EC, Mulder A, Rietman J S, Veltink PH (2011) Vibrotactile stimulation of the upper leg: Effects of location, stimulation method and habituation. Conf Proc IEEE Eng Med Biol Soc 2011: 1668-1671.

57. Rusaw D, Hagberg K, Nolan L, Ramstrand N (2012) Can vibratory feedback be used to improve postural stability in persons with transtibial limb loss? J Rehabilitative Research Development 49(8): 1239-1254.

58. Pailla T, Jiang W, Dichter B, Chang EF, Gilja V (2016) ECoG data analyses to inform closed-loop BCI experiments for speech-based prosthetic applications. Conference Proceedings in Medicine and Biology Society 2016: 5713-5716.

59. Yekta S, Lückhoff A, Ristić D, Lampert F, Ellrich J (2012) Impaired somatosensation of the tongue mucosa of smokers. Clinical Oral Investigations 16(1): 39-44.

60. Snyder D, Bartoshuk L (2016) Oral sensory nerve damage: Causes and consequences. Reviews in Endocrine and Metabolic Disorders 17(2): 149-158.

61. Bjerg L, Hulman A, Charles M, Jørgensen M, Witte D (2018) Clustering of microvascular complications in Type 1 diabetes mellitus. Journal Diabetes Complications 32(4): 393-399.

62. Gescheider GA, Edwards RR, Lackner EA, Bolanowski SJ, Verrillo RT (1996) The effects of aging on information-processing channels in the sense of touch: III. Differential sensitivity to changes in stimulus intensity. Somatosensory Motor Research 13(1): 73-80.

63. Stevens JC, Cruz LA (1996) Spatial acuity of touch: ubiquitous decline with aging revealed by repeated threshold testing. Somatosensory Motor Research 13(1): 1-10.

64. Oh H, Custead R, Wang Y, Barlow SM (2017) Neural encoding of saltatory pneumotactile velocity in human glabrous hand. PLoS ONE 12(8): e0183532. 
ISSN: 2574-1241

DOI: 10.26717/BJSTR.2019.23.003899

Steven M Barlow. Biomed J Sci \& Tech Res

(CC) This work is licensed under Creative Commons Attribution 4.0 License

Submission Link: https://biomedres.us/submit-manuscript.php

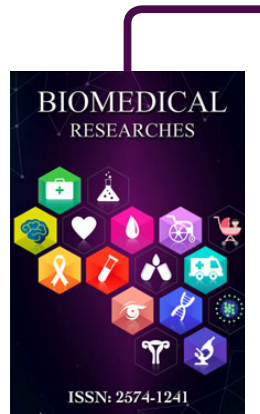

Assets of Publishing with us

- Global archiving of articles

- Immediate, unrestricted online access

- Rigorous Peer Review Process

- Authors Retain Copyrights

- Unique DOI for all articles 